PROCEEDINGS OF THE AMERICAN MATHEMATICAL SOCIETY

Volume 127, Number 3, March 1999, Pages 637-646

S 0002-9939(99)04534-7

\title{
SYMMETRIES OF ACCOLA-MACLACHLAN AND KULKARNI SURFACES
}

\author{
S. A. Broughton, E. BujAlance, A. F. COSTA, J. M. GAMBOA, AND \\ G. GROMADZKI
}

(Communicated by Albert Baernstein II)

\begin{abstract}
For all $g \geq 2$ there is a Riemann surface of genus $g$ whose automorphism group has order $8 g+8$, establishing a lower bound for the possible orders of automorphism groups of Riemann surfaces. Accola and Maclachlan established the existence of such surfaces; we shall call them Accola-Maclachlan surfaces. Later Kulkarni proved that for sufficiently large $g$ the Accola-Maclachlan surface was unique for $g=0,1,2 \bmod 4$ and produced exactly one additional surface (the Kulkarni surface) for $g=3 \bmod 4$. In this paper we determine the symmetries of these special surfaces, computing the number of ovals and the separability of the symmetries. The results are then applied to classify the real forms of these complex algebraic curves. Explicit equations of these real forms of Accola-Maclachlan surfaces are given in all but one case.
\end{abstract}

\section{INTRODUCTION}

In the 1960's Accola [1] and Maclachlan [12] proved independently that for every $g \geq 2$ there is a Riemann surface $X_{g}$ (Accola-Maclachlan surface) of genus $g$ whose automorphism group has order $8 g+8$, by explicitly giving the equation of the surface, $w^{2}=z^{2 g+2}-1$, and calculating its automorphism group. This result is interesting in that it is the largest order of an automorphism group that can be uniformly constructed for every $g$. This extended a result of Wiman in the last century in which he explicity constructed a surface (Wiman surface) of genus $g$, $w^{2}=z^{2 g+1}-1$ with an automorphism of maximal order $4 g+2$. Much later, Kulkarni [8] considered the question of uniqueness of the surfaces, i.e., whether they were the only surfaces of genus $g$ with an automorphism group of order $8 g+8$. Kulkarni demonstrated uniqueness for $g=0,1,2 \bmod 4$ and $g$ sufficiently large. For $g=3 \bmod 4$ and sufficiently large $g$ he also proved, that in addition to the Accola-Maclachlan surface, there is exactly one other surface of genus $g$ whose automorphism group has order $8 g+8$, though he did not give explicit equations for the surfaces. We shall call this surface the Kulkarni surface denoted by $Y_{g}$. He also showed that the conformal automorphism group of $X_{g}$ has the following

Received by the editors November 15, 1995 and, in revised form, June 5, 1997.

1991 Mathematics Subject Classification. Primary 14H45, 14E09, 14 H30.

The second and third authors were partially supported by DGICYT PB 95-0017 and CEECHRX-CT93-0408.

The fourth author was partially supported by DGICYT PB 95-0354 and CEE-CHRX-CT930408.

The fifth author was partially supported by the Pedagogical University of Bydgoszcz. 
presentation $\left\langle a, b \mid a^{2(g+1)}, b^{4},(a b)^{2}, a b^{2} a^{-1} b^{2}\right\rangle$ and the one of $Y_{g}$ has the following presentation $\left\langle a, b \mid a^{2(g+1)}, b^{4},(a b)^{2}, b^{2} a b^{2} a^{g}\right\rangle$.

In this paper we prove that both the Accola-Maclachlan and the Kulkarni surfaces are symmetric and we determine their symmetries. The results are given in the Table in Theorem 3.2. Our purpose is to not only examine the symmetries themselves, but also to determine the real forms of these two special families of Riemann surfaces. Before proceeding, let us recall a few facts about symmetries and real forms. Let $X$ be a surface of genus $g$. The group of conformal automorphisms of $X$ is denoted by $\operatorname{Aut}^{+}(X)$, and the group of all automorphisms of $X$, both conformal and anti-conformal, is denoted by $\operatorname{Aut}^{ \pm}(X)$. A symmetry $\sigma$ of $X$ is an anti-conformal, involutary automorphism, i.e., an element of $\operatorname{Aut}^{ \pm}(X) \backslash \operatorname{Aut}^{+}(X)$ of order 2. The fixed point set $X_{\sigma} \subset X$ of a symmetry $\sigma$ is a finite number of disjoint curves diffeomorphic to circles, which are called ovals of the symmetry. We denote by $k(X, \sigma)$ the number of ovals of $\sigma$. Define the symbol $\varepsilon(X, \sigma)$ to be 0 if $X \backslash X_{\sigma}$ is not connected - (we say that $\sigma$ is separating) - and 1 otherwise - (we say that $\sigma$ is non-separating). To abbreviate we define the species of $(X, \sigma)$ as

$$
\operatorname{sp}(X, \sigma)= \begin{cases}+k(X, \sigma) & \text { if } \varepsilon(X, \sigma)=0 \\ -k(X, \sigma) & \text { if } \varepsilon(X, \sigma)=1\end{cases}
$$

Two pairs $(X, \sigma)$ and $(Y, \tau)$ are said to be topologically equivalent if there exists a homeomorphism $h: X \rightarrow Y$ such that $\tau h=h \sigma$. Weichold [16] proved that $(X, \sigma)$ and $(Y, \tau)$ are topologically equivalent if and only if $X$ and $Y$ have the same genus and $\operatorname{sp}(X, \sigma)=\operatorname{sp}(Y, \tau)$. Later, Harnack [5] proved that given a triple of integers $(g, k, \varepsilon)$ there exists a pair $(X, \sigma)$ such that $g$ is the genus of $X, k=k(X, \sigma)$ and $\varepsilon=\varepsilon(X, \sigma)$ if and only if

$$
1 \leq k+\varepsilon \leq g+1 ; k \equiv g+1 \quad \bmod (2-\varepsilon) .
$$

Clearly, two conjugate symmetries $\sigma$ and $\tau$ on $X$ with respect to $\operatorname{Aut}^{ \pm}(X)$ have the same species since the pairs $(X, \sigma)$ and $(X, \tau)$ are topologically equivalent. The symmetry type of a surface is the list of species of all conjugacy classes of symmetries occurring in $\operatorname{Aut}^{ \pm}(X)$.

There is a 1-1 correspondence between symmetries and real forms of algebraic curves. In particular, if an algebraic curve can be defined by polynomial equations with real coefficients, then complex conjugation defines a symmetry of the corresponding Riemann surface. The ovals of a symmetry are the connected components of the corresponding real form. A real form is separating if it disconnects its complexification, and non-separating otherwise.

We shall determine the symmetry types of the Accola-Maclachlan surfaces and the Kulkarni surfaces, computing the number of ovals and separability of the symmetries. The results are then applied to determine the real forms of these complex algebraic curves. Explicit equations of the real forms of the curves are given in all cases but one.

\section{Number OF OVALS}

From the Riemann uniformization theorem, every compact Riemann surface $X$ of genus $g \geq 2$ can be represented as the quotient $H / \Gamma$ of the upper half complex plane $H$ under the action of a fuchsian surface group $\Gamma$. Thus there exists a Fuchsian group $\Delta$ containing $\Gamma$ as a normal subgroup such that $G=\operatorname{Aut}^{+}(X)=\Delta / \Gamma$. Although the method we are going to outline works in a more general setting, as in 
[4], for $X=X_{g}$ or $Y_{g}$ it is sufficient to analyze what happens when $\Delta$ is generated by three elliptic elements of even distinct orders $k, l$ and $m$ (actually in our case $k=2(g+1), l=4, m=2)$.

The unique NEC (non-euclidean crystallographic) group $\Lambda$ containing $\Delta$ as a subgroup of index 2 has the presentation

$$
\Lambda=\left\langle c_{0}, c_{1}, c_{2} \mid c_{0}^{2}, c_{1}^{2}, c_{2}^{2},\left(c_{0} c_{1}\right)^{k},\left(c_{1} c_{2}\right)^{l},\left(c_{0} c_{2}\right)^{m}\right\rangle
$$

and we shall refer to $c_{0}, c_{1}$ and $c_{2}$ as the canonical reflections of $\Lambda$.

From the presentations it is trivial to see that the assignment $a \mapsto a^{-1}, b \mapsto b^{-1}$ induces an automorphism

$$
\varphi: G \rightarrow G
$$

and so applying the criterion of Singerman [15] $X$ is symmetric. This is equivalent to saying that $\Gamma$ is a normal subgroup of $\Lambda$, and precisely, the symmetries on $X$ are the involutions $x \Gamma \in \Lambda / \Gamma=\widetilde{G}, x \in \Lambda \backslash \Delta$. We shall see later that the images $\theta\left(c_{i}\right)=\sigma_{i}, i=0,1,2$, of the $c$-reflections under the canonical projection $\theta: \Lambda \rightarrow \Lambda / \Gamma$ are not conjugate in $\widetilde{G}$. Since all reflections in $\Lambda$ are conjugate to one of the $c$-reflections and each symmetry $\sigma$ in $X$ with a non-empty set of fixed points $X_{\sigma}$ is the image $\sigma=\theta(c)$ of some reflection $c \in \Lambda$ (see [4]), classifying all symmetries $\sigma$ with $X_{\sigma}$ non-empty reduces to computing the $\operatorname{species} \operatorname{sp}\left(X, \sigma_{i}\right), i=0,1,2$. In this section we calculate $k\left(X, \sigma_{i}\right)=k_{i}$. If we represent the subgroup $\left\langle\sigma_{i}\right\rangle$ as $\Lambda_{i} / \Gamma$ for some group $\Lambda_{i} \subset \Lambda$ it follows that $k_{i}$ is the number of boundary components of the Klein surface $X /\left\langle\sigma_{i}\right\rangle=H / \Lambda_{i}$. In other words, $k_{i}$ is the number of conjugacy classes of reflections in $\Lambda_{i}$. If $C_{i}$ is the preimage under $\theta$ of the centralizer $C\left(\widetilde{G}, \sigma_{i}\right)$ of $\sigma_{i}$ in $\widetilde{G}$, the set of all reflections of $\Lambda_{i}$ is

$$
R_{i}=\left\{c_{i}^{w}=w c_{i} w^{-1}: w \in C_{i}\right\} .
$$

Now given $u, v \in C_{i}$, the reflections $c_{i}^{u}$ and $c_{i}^{v}$ are conjugate in $\Lambda_{i}$ if and only if $u^{-1} \gamma v \in C\left(\Lambda, c_{i}\right)$ for some $\gamma \in \Lambda_{i}$. On the other hand, $u^{-1} \gamma v=\left(u^{-1} v\right)\left(v^{-1} \gamma v\right)$. So as $C_{i}$ normalizes $\Lambda_{i}$, we see that $u^{-1} \gamma v \in C\left(\Lambda, c_{i}\right)$ if and only if $u^{-1} v \in C\left(\Lambda, c_{i}\right) \Lambda_{i}=$ $C\left(\Lambda, c_{i}\right) \Gamma$. Hence we get

$$
\left.k_{i}=\left[C_{i}: C\left(\Lambda, c_{i}\right) \Gamma\right]=\left[C_{i} / \Gamma: C\left(\Lambda, c_{i}\right) \Gamma / \Gamma\right]=\left[C\left(\widetilde{G}, \sigma_{i}\right)\right): \theta\left(C\left(\Lambda, c_{i}\right)\right)\right] .
$$

In particular, for $i=0$ it is easy to prove that

$$
C\left(\Lambda, c_{0}\right)=\left\langle c_{0}\right\rangle \oplus\left(\left\langle\left(c_{0} c_{1}\right)^{k / 2}\right\rangle *\left\langle\left(c_{0} c_{2}\right)^{m / 2}\right\rangle\right)
$$

and so

$$
k_{0}=\frac{\# C\left(\widetilde{G}, \sigma_{0}\right)}{4 \#\left(\left(\sigma_{0} \sigma_{1}\right)^{k / 2}\left(\sigma_{0} \sigma_{2}\right)^{m / 2}\right)}
$$

and similar formulae can be found for $k_{1}$ and $k_{2}$ (see [4] and [15]).

Let us attack the explicit computations in case $X=X_{g}$. Here we have

$$
\theta: \Lambda \rightarrow \Lambda / \Gamma=\operatorname{Aut}^{+}(X) \times_{\varphi} Z_{2}=\langle a, b\rangle \times_{\varphi}\langle t\rangle
$$

defined by

$$
\theta\left(c_{0}\right)=a t, \theta\left(c_{1}\right)=t, \theta\left(c_{2}\right)=t b,
$$

where $\varphi$ is defined in (3).

To apply (6) we must check that $\sigma_{0}, \sigma_{1}, \sigma_{2}$ are not conjugate in $\widetilde{G}$. In fact, each element $x \in \widetilde{G}$ can be written in a unique way as $x=a^{r} b^{s} t^{\mu}$ with $0 \leq r \leq$ 
$2 g+1,0 \leq s \leq 3,0 \leq \mu \leq 1$. Hence, $x t x^{-1}=a^{2 r} b^{2 s} t$ as $b^{2}$ commutes with $a$. So $\sigma_{0}=a t$ and $\sigma_{1}=t$ are not conjugate. Analogously we check the other two cases. Let us calculate the order of $C\left(\widetilde{G}, \sigma_{0}\right)$. The element $x \in \widetilde{G}$ above commutes with $\sigma_{0}$ if and only if either

$$
a=a^{r} b^{s} a b^{s} a^{r}, \mu=0 \quad \text { or } a=a^{r} b^{s} a^{-1} b^{s} a^{r}, \mu=1 .
$$

In both cases we have eight solutions. In the first case we have

$$
s=0,2 ; r=0, g+1 \text { and } s=1,3 ; r=1, g+2 .
$$

while in the the second case we have

$$
s=0,2 ; r=1, g+2 \text { and } s=1,3 ; r=0, g+1 .
$$

Consequently, $\# C\left(\widetilde{G}, \sigma_{0}\right)=16$. On the other hand, $\left(\sigma_{0} \sigma_{1}\right)^{k / 2}\left(\sigma_{0} \sigma_{2}\right)^{m / 2}=a^{g+2} b$ has order 2 for odd $g$ and order 4 for even $g$. Hence from (6), $k_{0}=2$ for odd $g$ and $k_{0}=1$ for even $g$. Similarly we prove that $k_{1}=1$ and $k_{2}=g+1$.

We still have to determine how many conjugacy classes of symmetries without fixed points exist. Such symmetries are elements of order $2, y=a^{r} b^{s} t \in \widetilde{G}$ which are not conjugate to $t, a t, t b$ and it is easy to see that there is exactly one class of such elements for even $g$ represented by $a b^{2} t$, and two classes represented by $a b^{2} t$ and $a^{g+1} b t$ if $g$ is odd.

Concluding, for odd $g, X_{g}$ has five classes of symmetries: two without fixed points, and three $\sigma_{0}, \sigma_{1}, \sigma_{2}$ with 2,1 and $g+1$ ovals, respectively. For even $g$ there are four conjugacy classes of symmetries: one without fixed points, two with one oval and one more with $g+1$ ovals.

The analysis of the symmetries of $Y_{g}$ for $g \equiv 3 \bmod 4$ follows the same technical lines. There are three classes of symmetries $\sigma_{0}, \sigma_{1}, \sigma_{2}$ with fixed points having 2,1 and $(g+1) / 4$ ovals and if $g \equiv 3 \bmod 8$ there is exactly one more class without fixed points.

\section{Separability character of the symmetries}

Let $\sigma$ be a symmetry on $X=X_{g}$ or $Y_{g}$. If $X_{\sigma}$ is empty, then $\sigma$ is non-separating. Thus, in what follows we are going to compute $\varepsilon\left(X, \sigma_{i}\right), i=0,1,2$, with the notation of the preceding sections. It follows from (1) that $\varepsilon=0$ if $k=g+1$, and $\varepsilon=1$ when $k=1$ and $g$ is odd. Furthermore since $(g+1) / 4$ is odd for $g \equiv 3 \bmod 8$, we conclude that $\varepsilon=1$ in the case $k=\frac{g+1}{4}, X=Y_{g}, g \equiv 3 \bmod 8$. Consequently

$$
\operatorname{sp}(X, \sigma)= \begin{cases}+(g+1) & \text { if }(X, \sigma)=\left(X_{g}, \sigma_{2}\right), \\ -1 & \text { if } \sigma=\sigma_{1} \text { except if } X=X_{g} \text { and } g \text { is even, } \\ -(g+1) / 4 & \text { if }(X, \sigma)=\left(Y_{g}, \sigma_{2}\right) \text { and } g \equiv 3 \bmod 8 .\end{cases}
$$

On the other hand, surfaces admitting a symmetry $\sigma_{2}$ with species $+(g+1)$ have been studied by Natanzon in [14] and by Bujalance and Costa in [3] and it follows directly from Theorem 3.3 in the latter paper that

$$
\operatorname{sp}\left(X_{g}, \sigma_{0}\right)=+2 \text { if } g \text { is odd. }
$$

To calculate $\varepsilon(X, \sigma)$ in the remaining cases we use two distinct strategies. First we prove:

$$
\operatorname{sp}(X, \sigma)= \begin{cases}-1 & \text { if }(X, \sigma)=\left(X_{g}, \sigma_{1}\right) \text { and } g \text { is even, } \\ -(g+1) / 4 & \text { if }(X, \sigma)=\left(Y_{g}, \sigma_{2}\right) \text { and } g \equiv 7 \quad \bmod 8 .\end{cases}
$$


For this purpose we need the following result which combines Theorem 1 in [11] and Lemma 2.8 in [2]:

Proposition 3.1. Let $G=A u t^{+}(X), X=X_{g}$ or $Y_{g}$, with the presentation of the introduction. Let $\sigma, \tau$ be two commuting symmetries on $X$. Let us define for each $x \in G$ the integer $\delta_{x}$ to be either 1 if $h=\sigma \tau$ is conjugate in $G$ to some power of $x$ and 0 otherwise. Then, if $\sigma$ is separating, the following inequality holds:

$$
\# C(G, h)\left(\frac{\delta_{a}}{2 g+2}+\frac{\delta_{b}}{4}+\frac{\delta_{a b}}{2}\right) \leq 2 k(X, \sigma) .
$$

Now if we apply this with $X=X_{g}, \sigma=\sigma_{1}, \tau=\sigma_{2} \sigma_{1} \sigma_{2}^{-1}$, then $h=b^{2}$ has order $2, \delta_{a}=\delta_{a b}=0, \delta_{b}=1$ and $\# C(G, h)=8(g+1)$. Hence, if $\sigma_{1}$ is separating we get from (11) $g+1 \leq k\left(X_{g}, \sigma_{1}\right) \leq 1$, a contradiction. Analogously, if $X=Y_{g}, \sigma=\sigma_{2}$, and $\tau=\sigma_{1} \sigma_{2} \sigma_{1}^{-1}$ we get again $h=b^{2}$ has order $2, \delta_{a}=\delta_{a b}=0, \delta_{b}=1$ and now $\# C(G, h)=4(g+1)$. Thus if $\sigma_{2}$ is separating, $(g+1) / 4=k\left(Y_{g}, \sigma_{2}\right) \geq(g+1) / 2$ which is absurd. This proves (10).

To finish we prove the following

$$
\operatorname{sp}\left(X_{g}, \sigma_{0}\right)=+1 \text { if } g \text { is even; } \operatorname{sp}\left(Y_{g}, \sigma_{0}\right)=-2 .
$$

In this case we apply a different approach due to Hoare and Singerman [6]. Let $X$ be either $X_{g}$ or $Y_{g}$ and with the notation in Section 2 let $\Gamma$ be a Fuchsian surface group uniformizing $X$. Let us consider the subgroup $K=\left\langle\sigma_{0}, \sigma_{1}\right\rangle$ of $\widetilde{G}=\operatorname{Aut}^{ \pm}(X)$, which is isomorphic to the dihedral group $D_{2 g+2}$ of $4 g+4$ elements. Then $K$ can be written as $\Gamma^{\prime} / \Gamma$ for some NEC-group $\Gamma^{\prime}$ whose signature

$$
\sigma\left(\Gamma^{\prime}\right)=(0 ;+;[-] ;\{(2 g+2,2 g+2, g+1)\})
$$

can be easily computed by the Riemann-Hurwitz formula (see e.g. [13] pg. 438). Let $\rho_{0}, \rho_{1}, \rho_{2}$ be a set of canonical reflections generating $\Gamma^{\prime}$ such that the natural projection $\Phi: \Gamma^{\prime} \rightarrow K$ maps $\rho_{j} \mapsto \sigma_{j}, j=0,1$. It is easy to see that

$$
\Phi\left(\rho_{2}\right)= \begin{cases}\sigma_{0}\left(\sigma_{1} \sigma_{0}\right)^{2 g} & \text { if } X=X_{g} \\ \sigma_{0}\left(\sigma_{1} \sigma_{0}\right)^{g-1} & \text { if } X=Y_{g}\end{cases}
$$

Following the quoted result in [6], the symmetry $\sigma_{0}$ is separating if and only if the Schreier graph $\mathcal{S}$ of the set of cosets $K /\left\langle\sigma_{0}\right\rangle$ corresponding to the system of generators $\left\{\Phi\left(\rho_{j}\right): j=0,1,2\right\}$ is bipartite. Assume now $X=X_{g}$. The $2 g+2$ classes in $K /\left\langle\sigma_{0}\right\rangle$ are denoted by $K /\left\langle\sigma_{0}\right\rangle=\{[i] \quad: \quad 0 \leq i \leq 2 g+1\}$, where $[i]=\left\{\left(\sigma_{0} \sigma_{1}\right)^{i},\left(\sigma_{0} \sigma_{1}\right)^{i} \sigma_{0}\right\}$. To produce the graph $\mathcal{S}$ it is necessary to know how each $\Phi\left(\rho_{j}\right), j=0,1,2$, links two vertices of $K /\left\langle\sigma_{0}\right\rangle$. But it is easy to check that the action of $\Phi\left(\rho_{j}\right)$ on the vertices is given by

$$
\Phi\left(\rho_{j}\right)([i])=[2 g+2-i-j], 0 \leq i \leq 2 g+1,0 \leq j \leq 2 .
$$

Then the Schreier graph $\mathcal{S}$ (with the loops deleted) admits a bipartition

$$
V_{1}=\{[i]: 0 \leq i \leq g\} ; V_{2}=\{[i]: g+1 \leq i \leq 2 g+1\}
$$

of the set of vertices of $\mathcal{S}$, and so, $\sigma_{0}$ is separating in this case. In a similar way it is proved that $\sigma_{0}$ is not separating if $X=Y_{g}$. We summarize our results in the following Theorem. 
Theorem 3.2. The symmetry types of the Accola-Maclachlan surface and the Kulkarni surface are as in the following table:

\begin{tabular}{||c|c|c|c|c||}
\hline \hline$X$ & $g$ & $\sigma_{0}$ & $\sigma_{1}$ & $\sigma_{2}$ \\
\hline \hline$X_{g}$ & odd & +2 & -1 & $+(g+1)$ \\
\hline$X_{g}$ & even & +1 & -1 & $+(g+1)$ \\
\hline$Y_{g}$ & $\equiv 7 \bmod 8$ & -2 & -1 & $-(g+1) / 4$ \\
\hline$Y_{g}$ & $\equiv 3 \bmod 8$ & -2 & -1 & $-(g+1) / 4$ \\
\hline \hline
\end{tabular}

\section{Real plane Curves RePRESEnting the Real Forms}

Let $N=g+1, P(z)=z^{2 N}-1$ and consider the complex plane curve

$$
C=\left\{(z, w) \in \mathbf{C}^{2}: w^{2}=P(z)\right\} .
$$

It follows from Theorem 3.2 that there exist three polynomials $Q_{i}(z) \in \mathbf{R}[z], i=$ $0,1,2$, such that each curve $C_{i}=\left\{(z, w) \in \mathbf{C}^{2}: w^{2}=Q_{i}(z)\right\}$ is C-birationally isomorphic to $\mathbf{C}$, and their real parts $C_{i}(\mathbf{R})=\left\{(z, w) \in \mathbf{R}^{2}: w^{2}=Q_{i}(z)\right\}$ have the species of the symmetries $\sigma_{i}, i=0,1,2$. Let $X_{i}$ denote a projective, smooth, real model of $C_{i}(\mathbf{R})$, i.e, a smooth, projective real algebraic curve, R-birationally isomorphic to $C_{i}(\mathbf{R})$. Let us denote by $X_{g}$ a (common) nonsingular complexification of $X_{i}$. We must prove that:

4.1. $X_{1}$ and $X_{g}-X_{1}$ are connected.

4.2. $X_{0}$ is connected if $N$ is odd and it has two connected components if $N$ is even. In both cases $X_{g}-X_{0}$ is not connected.

4.3. $X_{2}$ has $N$ connected components, thus $X_{g}-X_{0}$ is not connected.

In order to construct the polynomials $Q_{i}$, we examine the automorphism group of $X_{g}$. Recall that a presentation of the automorphism group $G$ of $X_{g}$ is

$$
G=\left\langle a, b \mid a^{2 N}, b^{4},(a b)^{2}, a b^{2} a^{-1} b^{2}\right\rangle .
$$

We can explicitly determine the generators $a$ and $b$, in fact, since $C$ and $X_{g}$ are C-birationally isomorphic, $G$ is isomorphic to the group of automorphisms of the function field $k_{g}=\left(\mathbf{C}[z, w] / w^{2}-P(z)\right)_{(0)}$. It is easy to see that if $\xi=e^{\frac{2 \pi i}{2 N}}$, the maps $a, b: k_{g} \rightarrow k_{g}$ defined by

$$
a(z)=\xi z, a(w)=-w ; b(z)=\frac{1}{z}, b(w)=\frac{i w}{z^{N}} ; a, b \mid \mathbf{C}=\text { identity },
$$

are isomorphisms satisfying the required relations.

Moreover, complex conjugation on $C$ corresponds to the $\mathbf{R}$-isomorphism $\sigma$ of $k_{g}$ defined by $\sigma(z)=z, \sigma(w)=w, \sigma(i)=-i$. We can explicitly compute the action of $a, b$ and $\sigma$ on $z, w$ and $i$ and it follows that $\sigma a \sigma=a^{-1} ; \sigma b \sigma=b^{-1} ; a \sigma, b \sigma$ are symmetries of $X_{g}$. Observe the similarity with the equalities $t a t^{-1}=a^{-1}$; $t b t^{-1}=b^{-1}$; at $=\theta\left(c_{0}\right), t b=\theta\left(c_{2}\right)$ from Section 2. For technical reasons we have chosen $b \sigma$ instead of $\sigma b=\sigma(b \sigma) \sigma^{-1}$, but both are conjugates. We will see that $\sigma, a \sigma$ and $b \sigma$ are representatives of the conjugacy classes of symmetries with fixed points in $X_{g}$. This will allow us to calculate the polynomials $Q_{i}$. In fact we prove that, with the notations of Section $3, \sigma \equiv \sigma_{1}, a \sigma \equiv \sigma_{0}$ and $b \sigma \equiv \sigma_{2}$.

The fixed subfield of $\sigma$ is $\mathbf{R}(z, w)$, so we choose $Q_{1}(z)=P(z)$. Let us prove 4.1. The only point at infinity $(0: 1: 0)$ of $C=C_{1}$ is singular. From $w^{2}=z^{2 N}-1$ it follows $\left(\frac{w}{z^{N}}\right)^{2}=1-\frac{1}{z^{2 N}}$ and so $z=\infty$ if and only if $\frac{w}{z^{N}}= \pm 1$. Hence, $X_{g}$ is the affine curve $C_{1}$ with two points adjoined. We can represent $X_{g}$ as a glueing 


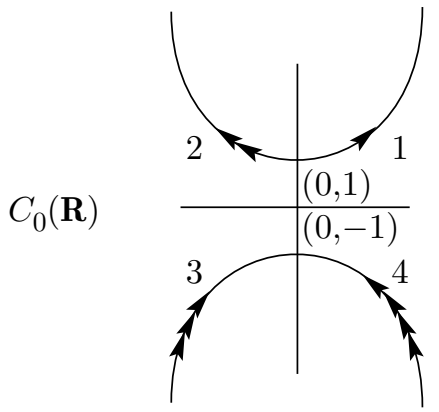

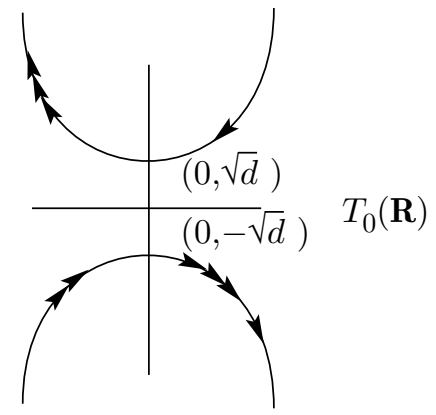

$N$ odd

FIGURE 1

by defining $t=\frac{1}{z}$, and $s=\frac{w}{z^{N}}$. Note that $s^{2}=1-t^{2 N}$ if $(z, w) \in C_{1}$. Then, $\varphi: C_{1} \rightarrow T_{1}=\left\{(t, s) \in \mathbf{C}^{2}: s^{2}=1-t^{2 N}\right\}$ defined by $\varphi(z, w)=\left(\frac{1}{z}, \frac{w}{z^{N}}\right)$ is a birational isomorphism preserving the real parts. Note that $t=0, s= \pm 1$ at the points at infinity, and so $X_{g}$ can be viewed as the glueing of $C_{1}$ and $T_{1}$ via $\varphi$, along the points $z \neq 0, t \neq 0$. Since $\varphi\left(C_{1}(\mathbf{R})\right)=T_{1}(\mathbf{R})$ and $C_{1}(\mathbf{R})$ does not intersect the line $(z=0)$, it follows that $X_{1}$ is connected because $T_{1}(\mathbf{R})$ is. Let us check that $X_{g}-X_{1}$ is also connected. The map $T_{1} \rightarrow \mathbf{C}:(t, s) \rightarrow t$ is a ramified double covering which ramifies at $t=\xi^{k}, k=0,1, \ldots, 2 N-1$, with two sheets $s_{\epsilon}: \mathbf{C} \rightarrow T_{1}: t \rightarrow\left(t, \epsilon \sqrt{1-t^{2 N}}\right), \epsilon= \pm 1$. The real points of $T_{1}$ project onto the closed interval $[-1,1] \subset \mathbf{R}$. Hence, using circles around the branching points, one can connect, avoiding points with $t \in[-1,1]$, points in the upper sheet with points in the bottom sheet, and so $X_{g}-X_{1}$ is connected.

We now construct $Q_{0}$. To do this, we determine two algebraically independent elements in $k_{g}$, fixed under the action of $a \sigma$. However, it is easy to see that $z_{1}=$ $(1+\xi) z$ and $w_{1}=i w$ are fixed by $a \sigma$ Since $k_{g}=\mathbf{C}(z, w)=\mathbf{C}\left(z_{1}, w_{1}\right)$, we can express the equation $w^{2}=z^{2 N}-1$ of $C$ in terms of $z_{1}$ and $w_{1}$. In fact $w=-i w_{1}$, and $z=\frac{z_{1}}{1+\xi}$. Thus, $-w_{1}^{2}=\frac{z_{1}^{2 N}}{(1+\xi)^{2 N}}-1$. By defining $d=\frac{-1}{(1+\xi)^{2 N}}$, we obtain $w_{1}^{2}=1+d z_{1}^{2 N}$. Note that $|\xi|=1$ and $\operatorname{Arg}(\xi)=\frac{\pi}{N}$, thus $\operatorname{Arg}(1+\xi)=\frac{\pi}{2 N}$ and $\operatorname{Arg}(1+\xi)^{2 N}=\pi$. Thus $d$ is a positive real number. We define $Q_{0}(z)=1+d z^{2 N}$. It is obvious that $C_{0}$ is $\mathbf{C}$-isomorphic to $C$.

We now prove 4.2. First we count the number of connected components of $X_{0}$. We represent $X_{g}$ as before as the glueing of $C_{0}$ with $T_{0}=\left\{(t, s) \in \mathbf{C}^{2}: s^{2}=d+t^{2 N}\right\}$ via the isomorphism $\varphi: C_{0} \rightarrow T_{0}:(z, w) \rightarrow\left(\frac{1}{z}, \frac{w}{z^{N}}\right)$, along the points $z \neq 0$, $t \neq 0$. This glueing behaves quite differently according to the parity of $N$. If $N$ is even, the intersection of $C_{0}(\mathbf{R})$ with the $j$-quadrant of $\mathbf{R}^{2}, j=1,2,3,4$, labelled counterclockwise, is identified with the intersection of $T_{0}(\mathbf{R})$ with the same quadrant. If $N$ is odd, the intersections with quadrants 2 and 3 are interchanged.

Considering Figure 1, one realizes that $X_{0}$ has two connected components if $N$ is even, since the points at infinity $(0,1)$ and $(0,-1)$ of $C_{0}(\mathbf{R})$ cannot be joined by a path, while $X_{0}$ is connected if $N$ is odd, because you can jump from the points in $T_{0}(\mathbf{R})$ lying in the second quadrant to the points in $C_{0}(\mathbf{R})$ lying on the third quadrant. 
To check that $X_{g}-X_{0}$ is disconnected, note that it is included in $C_{0}$, since $X_{0}$ contains the two points at infinity $(0, \pm \sqrt{d})$ of $T_{0}$. Now, consider two points $P_{0}=\left(z_{0}, w_{0}\right)$ and $P_{0}^{\prime}=\left(z_{0}^{\prime}, w_{0}^{\prime}\right)$ in $X_{g}-X_{0}$ such that $I_{m}\left(z_{0}\right)>0, I_{m}\left(z_{0}^{\prime}\right)<0$. These two points cannot be joined by a path in $X_{g}-X_{0}$. Otherwise, let $\gamma:[0,1] \rightarrow$ $X_{g}-X_{0}: s \rightarrow\left(\gamma_{1}(s), \gamma_{2}(s)\right)$ be such a path. Since $\gamma_{1}(0)$ and $\gamma_{1}(1)$ are complex numbers whose imaginary parts have opposite signs, there exists $s \in(0,1)$ such that $\gamma_{1}(s) \in \mathbf{R}$. Then, $1+\gamma_{1}(s)^{2 N}$ is a positive real number, whose square is $\gamma_{2}(s)$, and so $\gamma(s) \in X_{0}$, a contradiction. This finishes 4.2 .

We now construct the polynomial $Q_{2}$. As before, we try to find two elements in $k_{g}$, fixed by $b \sigma$ and algebraically independent over C. Note that $X_{2}$ has $N$ connected components, so $C_{2}(\mathbf{R})$, should have $N+1$ components, and for this to occur, $Q_{2}$ must have $2 N$ distinct real roots. So we transform the polynomial $P(z)=z^{2 N}-1$ with roots $\xi^{k}, k=0,1, \ldots, 2 N-1$, into $Q_{2}(z)$ with $2 N$ real roots, such that $C$ and $C_{2}$ are birationally isomorphic over $\mathbf{C}$. Consider the roots $\xi, 1,-1$ of $P$; we will transform these to the roots $0,1,-1$ of $Q_{2}$. The only Möbius transformation $\Phi: \mathbf{P}_{1}(\mathbf{C}) \rightarrow \mathbf{P}_{1}(\mathbf{C})$ which maps $\xi \rightarrow 0,1 \rightarrow 1$ and $-1 \rightarrow-1$ is $\Phi(z)=\frac{z-\xi}{1-\xi z}=z_{1}$, and this element is fixed by $b \sigma$, as can be easily checked!

It is evident that each $a_{k}=\Phi\left(\xi^{k}\right) \in \mathbf{R}, k=0,1, \ldots, 2 N-2$, and $a_{2 N-1}=$ $\Phi\left(\xi^{2 N-1}\right)=\infty$, and these numbers $a_{k}$ are pairwise distinct because $\Phi$ is injective. The inverse map of $\Phi$ is $\Psi(z)=\frac{z+\xi}{1+\xi z}$ and the rational function $R(z)=P(\Psi(z))$ vanishes at each $a_{k}$. But,

$$
R(z)=\left(\frac{z+\xi}{1+\xi z}\right)^{2 N}-1=\frac{(z+\xi)^{2 N}-(1+\xi z)^{2 N}}{(1+\xi z)^{2 N}}
$$

and $S(z)=(z+\xi)^{2 N}-(1+\xi z)^{2 N} \in \mathbf{C}[z]$ is a polynomial of degree $2 N-1$ which vanishes at $a_{0}, \ldots, a_{2 N-2}$, and whose leading coefficient is $2 N\left(\xi-\xi^{-1}\right)=$ $4 N i \sin \frac{\pi}{N}=\rho^{2}$, where $\rho=\eta e^{\frac{\pi i}{4}}$ and $\eta$ is a real positive number. Thus, $S(z)=$ $\rho^{2} Q_{2}(z)$, where

$$
Q_{2}(z)=\left(z-a_{0}\right) \ldots\left(z-a_{2 N-2}\right)
$$

is the polynomial we are looking for.

Note that the map $\alpha: C \rightarrow C_{2}:(z, w) \rightarrow\left(\Phi(z), \rho^{-1} w(1+\xi \Phi(z))^{N}\right)$ is a rational mapping for, if $(z, w) \in C$, then upon setting $\Phi(z)=u$, i.e., $\Psi(u)=z$, we obtain

$$
\begin{gathered}
w^{2}=z^{2 N}-1=\Psi(u)^{2 N}-1=P(\Psi(u))=R(u)=\frac{S(u)}{(1+\xi u)^{2 N}} \\
=\frac{\rho^{2}\left(u-a_{0}\right) \cdots\left(u-a_{2 N-2}\right)}{(1+\xi u)^{2 N}}
\end{gathered}
$$

i.e.,

$$
\left(u-a_{0}\right) \cdots\left(u-a_{2 N-2}\right)=\left[\rho^{-1} w(1+\xi u)^{N}\right]^{2} .
$$

In fact $\alpha$ is an isomorphism with inverse $\alpha^{-1}: C_{2} \rightarrow C:(u, v) \rightarrow\left(\Psi(u), \frac{\rho v}{(1+\xi u)^{N}}\right)$.

Thus $C_{2}$ is $\mathbf{C}$-isomorphic to $C$ and $X_{2}$ has $N$ components. We are in a position to prove also that $b \sigma$ represents the symmetry $\sigma_{2}$ of the previous section. In fact, $w_{1}=\rho^{-1} w\left(1+\xi z_{1}\right)^{N} \in k_{g}$ is fixed by $b \sigma$ because $b \sigma\left(w_{1}\right)=(\bar{\rho})^{-1} \frac{i w}{z^{N}}\left(1+\bar{\xi} z_{1}\right)^{N}$, and since $\bar{\xi}=\xi^{-1}$ and $\bar{\rho}=-i \rho$, we get

$$
b \sigma\left(w_{1}\right)=\frac{i}{\rho} \frac{i w}{z^{N}}\left(1+\frac{z_{1}}{\xi}\right)^{N}=\frac{w}{\rho} \frac{\left(\xi+z_{1}\right)^{N}}{z^{N}}=w_{1} .
$$


Hence the map $\alpha: C \rightarrow C_{2}$ is $\alpha(z, w)=\left(z_{1}, w_{1}\right)$ and $\mathbf{R}\left(z_{1}, w_{1}\right)$ is the fixed field of $b \sigma$, and so it represents $\sigma_{2}$.

For the sake of completeness we also consider the symmetries $a b^{2} \sigma$ and $a^{N} b \sigma$ (the last only for even $N$ ) which are fixed point free. The first fixes both $w$ and $u=(1+\xi) z$. From the equation $w^{2}=z^{2 N}-1$ ones gets $w^{2}=\frac{u^{2 N}}{(1+\xi)^{2 N}}-1$. We already noted that $\gamma=\frac{1}{(1+\xi)^{2 N}}$ is a real negative number, and so the curve $D_{1}=$ $\left\{(u, w) \in \mathbf{C}^{2}: w^{2}=\gamma u^{2 N}-1\right\}$ is $\mathbf{C}$-isomorphic to $C$ via the map $(z, w) \rightarrow(u, w)$, but the set $D_{1}(\mathbf{R})$ of real points of $D_{1}$ is empty.

Finally, if $N$ is even, $a^{N} b \sigma$ is a symmetry which is not a conjugate of $a b^{2} \sigma$, and it maps $z$ to $\frac{-1}{z}$ and $w$ to $\frac{i w}{z^{N}}$. Let $M=\frac{N}{2}$. Then $a^{N} b \sigma$ fixes $y=z-\frac{1}{z}, x=i\left(z+\frac{1}{z}\right)$ and $t=\frac{\lambda w}{z^{M}}$, where $\lambda=1+(-1)^{M} i$. Then $z=\frac{x+i y}{2 i}, \frac{1}{z}=\frac{x-i y}{2 i}$ and $w=\frac{t z^{M}}{\lambda}$. After substituting in $z^{2 N}-1=w^{2}$, we get $0=F(x, y, t)=t^{2}+\frac{1}{2^{N-2}} \sum_{j=0}^{M-1}(-1)^{j}$ $\left(\begin{array}{c}N \\ 2 j+1\end{array}\right) x^{N-1-2 j} y^{2 j+1}$. Also, $G(x, y)=x^{2}+y^{2}+4=0$. Thus $C$ is $\mathbf{C}$-isomorphic to $D_{2}=\left\{(x, y, t) \in \mathbf{C}^{3}: F(x, y, t)=G(x, y)=0\right\}$ and $D_{2}(\mathbf{R})$ is empty.

\section{ACKNOWLEDGMENT}

The authors thank the referee, who pointed out and corrected a mistake in Section 4 of the first version of this paper. The referee also suggested the use of generators of the field of functions fixed by a symmetry to find its real equation. This replaced an intuitive method which had been employed by the authors.

\section{REFERENCES}

1. Accola R. D. M.: On the number of automorphisms of a closed Riemann surface. Trans. Amer. Math. Soc. 131 (1968), 398-408. MR 36:5333

2. Broughton S. A., Bujalance E., Costa A. F., Gamboa J. M., Gromadzki G.: Symmetries of Riemann surfaces on which $\operatorname{PSL}(2, q)$ acts as Hurwitz automorphism group. J. Pure Appl. Alg. 106 (1996) 113-126. MR 97e:14043

3. Bujalance E., Costa A. F.: A combinatorial approach to the symmetries of $M$ and $M-1$ Riemann surfaces, Lecture Notes Series 173 London Math. Soc. (1992), 16-25. MR 93k:30075

4. Gromadzki G.: Groups of Automorphisms of Compact Riemann and Klein Surfaces. Habilitazionschrift. University Press WSP Bydgoszcz (1993).

5. Harnack A.: Über die Vieltheiligkeit der ebenen algebraischen Kurven. Math. Ann. 10 (1876), 189-199.

6. Hoare A. H. M., Singerman D.: Subgroups of plane groups. London Math. Soc. Lect. Note Series 71 (1982), 221-227. MR 85g:20061

7. Hurwitz A.: Über algebraische Gebilde mit eindeutigen Transformationen in sich. Math. Ann. 41 (1893), 402-442.

8. Kulkarni R. S.: A note on Wiman and Accola-Maclachlan surfaces. Ann. Acad. Sci. Fenn. 16 (1991), 83-94. MR 92j:30045

9. Macbeath A. M.: On a theorem of Hurwitz. Proc. Glasgow Math. Assoc. 5 (1961), 90-96. MR 26: 4244

10. Macbeath A. M.: Discontinuous groups and birational transformations. Proc. of Dundee Summer School, Univ. of St. Andrews (1961).

11. Macbeath A. M.: Action of automorphisms of a compact Riemann surface on the first homology. Bull. London Math. Soc. 5 (1973), 103-118. MR 47:8840

12. Maclachlan C.: A bound for the number of automorphisms of a compact Riemann surface. J. London Math. Soc. 44 (1969), 265-272. MR 38:4674

13. Nakagawa K.: On the orders of automorphisms of a closed Riemann surface. Pacific J. Math. 115 (1984), 435-443. MR 86a:30073

14. Natanzon S. M.: Automorphisms of the Riemann surface of an $M$-curve. Funktsional Anal. i Priloz. 12:3 (1978), 82-83. (Functional Anal. Appl. 12 (1978), 228-229.) MR 82b:14020 
15. Singerman D.: Automorphisms of compact non-orientable Riemann surfaces. Glasgow Math. J. 12 (1971), 50-59. MR 45:5347

16. Weichold G.: Über symmetrische Riemanns'che Flächen und die Periodicitäsmoduln der zugehörin Abel'schen Normalintegrale erster Gattung. Zeitschrift für Math. und Phys. 28 (1883), 321-351.

(S. A. Broughton) Department of Mathematics, Rose-Hulman Institute of Technology, Terre Haute, Indiana 47803

E-mail address: allen.broughton@rose-hulman.edu

(E. Bujalance and A. F. Costa) Departamento de Matematicas, Fund. Uned, 28040 MADRID, SPAIN

E-mail address: eb@mat.uned.es

E-mail address: acosta@mat.uned.es

(J. M. Gamboa) Departamento de Algebra, Universidad Complutense de Madrid, 28040 MADRID, SPAIN

E-mail address: jmgamboa@eucmax.sim.ucm.es

(G. Gromadzki) Instytut Matematyki WSP, Chodkiewicza 30, 85-064 Bydgoszcz, Poland

E-mail address: greggrom@mat.uned.es 